\title{
BCAM, the Basque Center for Applied Mathematics
}

Jean-Bernard Bru and Carlos Pérez (both University of the Basque Country and Basque Center for Applied Mathematics, Bilbao, Spain)

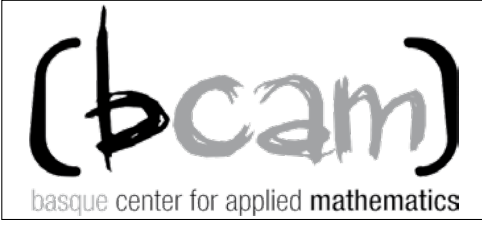

The Basque Center for Applied Mathematics (BCAM) is a worldclass research centre in the field of applied mathematics, located in Bilbao (Spain). Its main goal is promoting scientific and technological advances worldwide through interdisciplinary research in mathematics and training and attracting talented scientists.

Embedded in a multicultural environment, with more than 90 people from over 25 nationalities working there, BCAM is a young centre that provides the right atmosphere for research and promotes the creation of hardworking international and interdisciplinary teams.

\section{0 years of research at the frontiers of mathematics}

BCAM was founded 10 years ago, in September 2008, and Professor E. Zuazua was the founding scientific director. It was promoted by the Basque Government through Ikerbasque (Basque Foundation for Science) in the framework of the BERC (Basque Excellence Research Centers) network. The University of the Basque Country and Innobasque (Basque Innovation Agency) joined BCAM as founding members and the Biscay Regional Government joined later as an institutional member.

In 2013, BCAM was accredited for the first time as a "Severo Ochoa" centre of excellence by the State Research Agency, which has been part of the Spanish Ministry of Science, Innovation and Universities for four years. The accreditation was given to the centre for the second time during the 2017 call for proposals. This distinction, which recognises the international relevance of the scientific research carried out, as well as the global interest of the proposed work programme for the next four years, is given to the best research institutions in the world in their fields.

BCAM's team is led by Professor Luis Vega, a well renowned mathematician with an extensive professional career, who became BCAM's scientific director in 2013. Vega has been a full professor of mathematical analysis at the University of the Basque Country for 23 years and a visiting professor at several international universities. $\mathrm{He}$ is also a member of many international research institutes, a fellow of the American Mathematical Society, a member of the European Academy of Science and has recently been elected a member of the Spanish Royal Academy of Sciences (RAC). He received the Euskadi

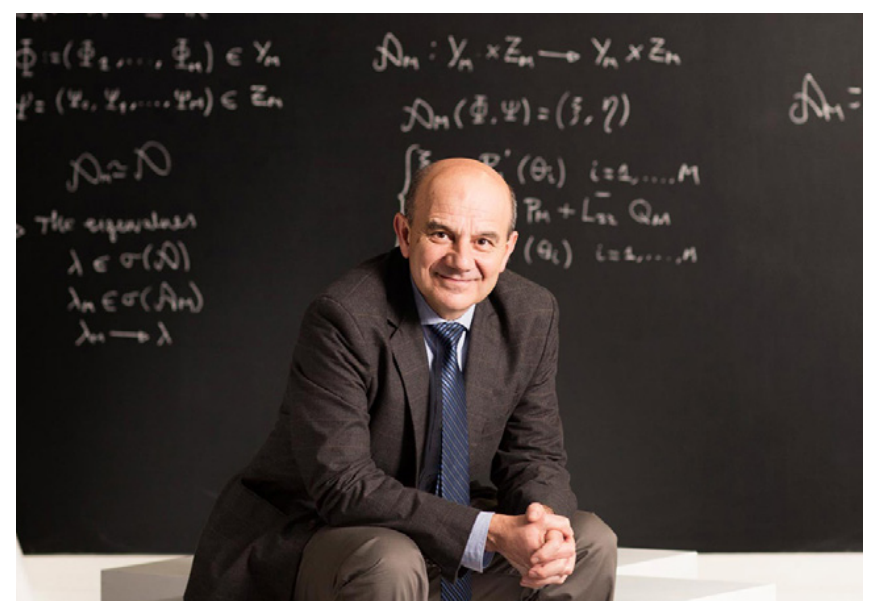

Luis Vega, BCAM's Scientific Director.

Research Prize 2012 and a European Research Council Advanced Grant in 2014 and was awarded with the 2015 Blaise Pascal Medal in Mathematics. Vega is also BCAM's representative at ERCOM, a committee under the EMS consisting of scientific directors of 26 European mathematical research centres.

According to him, making scientific progress and improving the social appreciation of mathematics will be BCAM's main goals on its 10th anniversary, as well as dissemination and training in this area. Regarding the day-to-day life at the centre, he claims that "it is essential that a research centre creates a healthy environment in which there is complicity among researchers and in which one can learn just by breathing" and he "strongly" believes that this is being achieved at BCAM.

\section{Research areas and projects}

The research carried out at the centre is oriented by three scientific platforms: Core in Applied Mathematics, Computational Mathematics and Applications of Mathematics.

The first platform, Core in Applied Mathematics, refers to research carried out on mathematical challenges at the frontier of knowledge. This corresponds to the purest mathematics and is somehow timeless because it faces the most important mathematical challenges of our time. Computational Mathematics, the centre's second platform, focuses on developing precise mathematical models and simulations, as well as numeric experiments, using powerful computational resources. Finally, the third platform, Applications of Mathematics, covers the techniques and methods that have real-life applications in industry and society in general. 


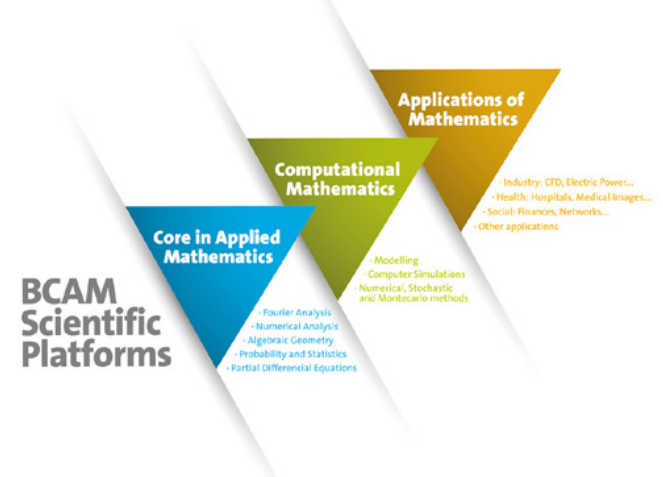

BCAM's Scientific Platforms.

This structure makes BCAM a multidisciplinary centre in which mathematics and computer science occupy more than $50 \%$ of the scientific production. The team at BCAM is distributed into five research areas covering various relevant fields of applied mathematics.

\section{Computational Mathematics (CM)}

This area is divided into three research lines: Simulation of Wave Propagation, CFD Microfluids \& Rheology and CFD Computational Technology. The researchers in this area work on new mathematical methods and robust numerical schemes and software to solve complex and large-scale, challenging, real-life problems on massively parallel computers. Some applications of their developments are the characterisation of the Earth's surface composition for $\mathrm{CO} 2$ sequestration and oil and gas extraction, computational fluid dynamics applied to medicine, meteorology, oceanography, aeronautics, naval architecture, acoustics and turbomachinery, and the tackling of several complex microflow problems in material, food and biomedical sciences.

\section{Mathematical Modelling with Multidisciplinary Applications (M3A)}

Many open challenges in life sciences modelling require efficient algorithms and robust supporting theories. The objective of the research lines included in the M3A group - Modelling and Simulation in Life and Materials Sciences, Mathematical Modelling in Biosciences, and Mathematical, Computational and Experimental Neuroscience - is the elaboration of novel theoretical and computational tools for efficient and detailed simulation of multi-scale complex systems describing real-life problems in biology, medicine, public health and society. In fact, BCAM is working on a new Neuroscience Laboratory that will develop new technologies based on mathematics and analyse large volumes of clinical data in collaboration with other research facilities. This laboratory will serve as an interface between neuroscientists and clinicians.

\section{Mathematical Physics (MP)}

At the interface between mathematics and physics is the so-called mathematical physics, which at BCAM is rep- resented by the research lines of Quantum Mechanics, Statistical Physics and Singularity Theory \& Algebraic Geometry. This group aims toward the mathematical understanding of theories of physics and the development of methods that could, in the future, be applied, for example, to quantum technologies, the forecast of wildland fire propagation to preserve natural heritage, cryptography and string theory.

\section{Analysis of Partial Differential Equations (APDE)}

This group uses PDE models to describe real-life phenomena efficiently. They explore and exploit the deep connections between partial differential equations, harmonic analysis, inverse problems and applied mathematics so as to describe the most diverse phenomena. The understanding of the fundamental principles that control the relevant phenomena in physics and biology could eventually become of use for scientists working in those fields. The group consists of three research lines: Linear and Non-linear Waves, Harmonic Analysis and Applied Analysis.

\section{Data Science (DS)}

The increase in data generation (big data) and problem sizes has made indispensable the development of new statistical and machine learning methods and algorithms for knowledge extraction and optimisation. The Data Science group at BCAM, divided into the Heuristic Optimisation, Applied Statistics and Machine Learning research lines, works on massive data and optimisation problems in the following areas: financial and social media, cybersecurity, marketing, medical domains (diagnosis and prognosis), genetics, environmental modelling, demography and biostatistics, logistics, and scheduling and planning.

Although BCAM is a multidisciplinary research centre, in the last period, a special effort has been made to encourage collaboration between different lines to foster synergies. In addition to the common research interests that some groups share, there are new initiatives in which teams that belong to different research areas participate together.

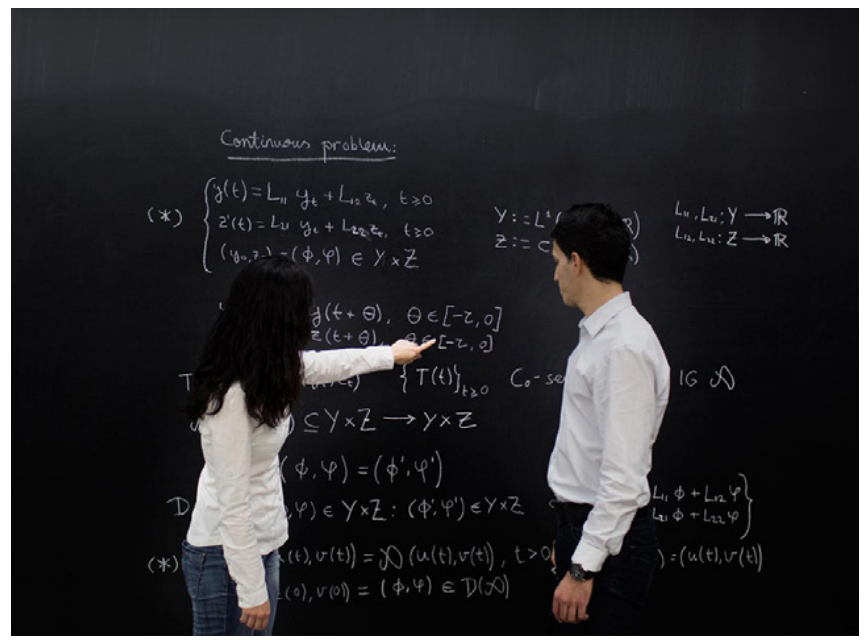

Researchers at BCAM's facilities. 
All the groups mentioned above work on highly competitive projects that range from top level projects, funded by the European Research Council (ERC) or the Marie Skłodowska-Curie Research and Innovation Staff Exchange, to research and development projects, funded by the Basque and Spanish Governments and even grants awarded by private companies.

\section{Knowledge transfer and dissemination}

Under the motto "Mathematics in the service of society", the Basque Center for Applied Mathematics aims to spread knowledge and technology in industry and society in general. It is critical for the centre to transfer the obtained research results to sectors such as biosciences, health, energy, advanced manufacturing, telecommunications and transport, including local, national and international entities.

Toward that goal, BCAM recently promoted its Knowledge Transfer Unit (KTU), a platform to develop mathematical solutions for scientific challenges based on real-life applications and collaborations with industry. These collaborations are developed in the form of strategic partnerships, R\&D\&I projects, joint positions, training courses, supervision of Master's and $\mathrm{PhD}$ students, organisation of dissemination activities, etc.

Some examples of the projects that have been developed by BCAM's KTU for private companies include the analysis of the oil price market, computational modelling for cardiac radiofrequency ablation and CFD simulation of the beam deposition process for a control laser device in additive manufacturing. Another interesting project that has just started is a collaboration with an athletics club football team to predict, prevent and manage injuries among the players through data science.

In line with its commitment to knowledge transfer, BCAM aims to promote the MSO \& Data Analysis Laboratory. The main goal of this platform will be to use applied mathematics to promote cooperation with other agents (social, industrial, clinical, etc.) by supporting them through the design of experiments, the simulation of diverse phenomena and the analysis of big amounts of data.

At the same time, BCAM has created and developed a very broad programme of scientific activities, addressed to all sectors of society and, particularly, to young scientists. In the last four years, the centre has organised more than 600 seminars and working groups, 90 workshops in collaboration with the main R\&D agents, 10 colloquiums and 100 courses. The aim of this comprehensive training programme is to transfer the specialised knowledge and results generated and nurtured at BCAM and communicate the importance of mathematical research and its applications to society.

Dissemination of mathematics for the general public and fostering scientific culture among citizens are also two important priorities of the centre. Researchers actively participate in dissemination activities in order to bring their research on mathematics closer to society.

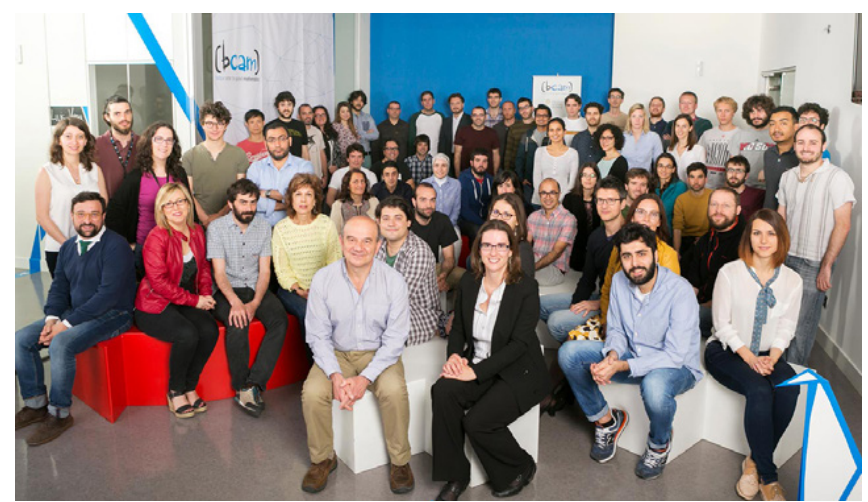

Part of the team working at BCAM.

\section{BCAM team}

From the administrative staff to the highly qualified and specialised international researchers, people within BCAM are the driving force of the centre. The current team is formed of more than 90 people, with over 25 nationalities represented, and the average age of the researchers is less than 35 .

Aware that young researchers are the heart of the centre, one of BCAM's main targets is to attract and provide them with competitive training so that they can develop their future careers in a successful way. With that purpose, BCAM has put into place several programmes to attract international researchers and students.

- The Visiting Fellow Programme: Every year, BCAM offers research opportunities for outstanding mathematicians from all over the world, for short-term and long-term visits.

- The Visitor Programme: Internationally-leading scientists are invited for short-term visits to disseminate and convey their ideas and recent and ongoing research through seminars and collaboration with BCAM researchers.

BCAM's facilities located in Bilbao.

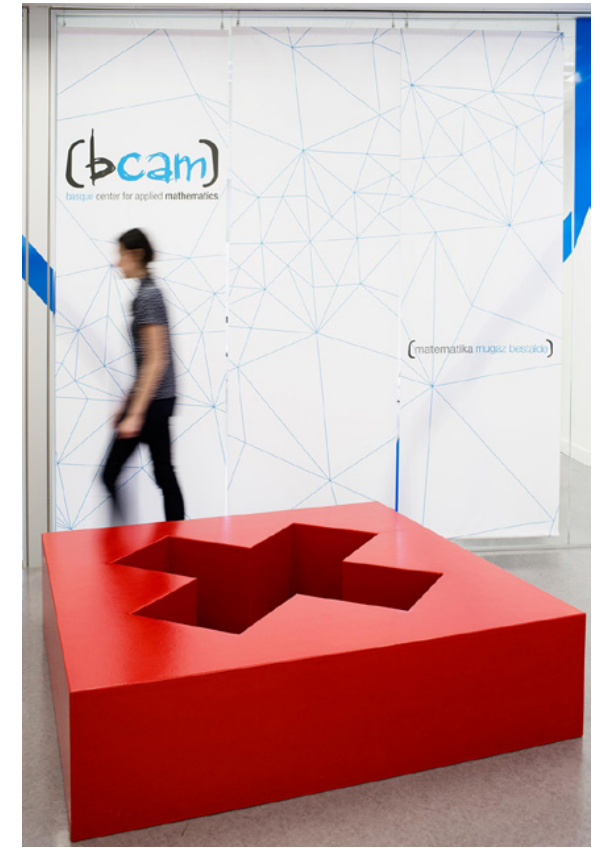


- The Internship Programme: Young people, appointed to BCAM in this programme, come from institutions where they are studying graduate or post-graduate courses and join BCAM as members of a specific team with a predefined area and tutor.

Finally, we must point out that BCAM signed its commitment to the European Researchers' Charter and the Code of Conduct for Researcher Recruitment in December 2008. In 2016, the centre was awarded the HR Logo related to "Human Resources Strategy For Researchers (HRS4R)", promoted by the European Commission.

Although the road ahead presents many challenges, such as seeking funding to consolidate research teams, developing new programmes and activities and strengthening links with international and local partners, BCAM will continue working to achieve outstanding scientific results to demonstrate that mathematics is a fundamental tool for the development of society.

If you would like to join us or find out more about BCAM's research, scientific activities and open positions, please visit www.bcamath.org, subscribe to our newsletter at https://bit.ly/2HshsXA or follow us on Twitter @BCAMBilbao.

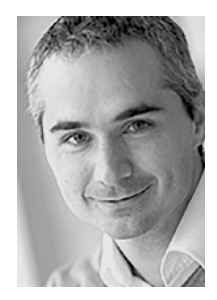

Jean-Bernard Bru is an Ikerbasque Research Professor at the Mathematics department of the University of the Basque Country (UPV/EHU) and at the Basque Center for Applied Mathematics (BCAM). He started his career as an independent researcher in 1999 with a PhD in mathematical physics from the Aix-Marseille University (France). The bulk of his research covers a scope from mathematical analyzes of the many-body problem to operator algebras, stochastic processes, differential equations, convex and functional analysis. He has participated in more than 64 conferences, performed at least 94 seminars and accomplished many research visits to universities across Armenia, Brazil, Europe and the USA.

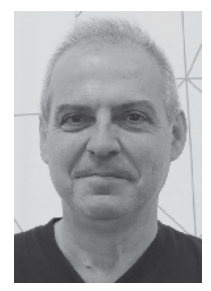

Carlos Pérez graduated in Mathematics at the Autonomous University of Madrid and obtained his PhD in Mathematics in 1989 at Washington University, Saint Louis (USA). He authored one book and more than 80 papers in International journals. He was full professor at the University of Seville until 2014 where he was appointed as Ikerbasque Research Professor at the University of the Basque Country and BCAM. 